\title{
EVENTUAL FINITE ORDER GENERATION FOR THE KERNEL OF THE DIMENSION GROUP REPRESENTATION
}

\author{
J. B. WAGONER
}

\begin{abstract}
The finite order generation problem (FOG) in symbolic dynamics asks whether every element in the kernel of the dimension group representation of a subshift of finite type $\left(X_{A}, \sigma_{A}\right)$ is a product of elements of finite order in the group $\operatorname{Aut}\left(\sigma_{A}\right)$ of homeomorphisms of $X_{A}$ commuting with $\sigma_{A}$. We study the space of strong shift equivalences over the nonnegative integers, and the first application is to prove Eventual FOG which says that every inert symmetry of $\sigma_{A}$ is a product of finite order homeomorphisms of $X_{A}$ commuting with sufficiently high powers of $\sigma_{A}$. Then we discuss the relation of FOG to Williams' lifting problem (LIFT) for symmetries of fixed points. In particular, either FOG or LIFT is false. Finally, we also discuss $p$-adic convergence and other implications of Eventual FOG for gyration numbers.
\end{abstract}

\section{INTRODUCTION}

Let $\operatorname{Aut}_{0}\left(\sigma_{A}\right)$ denote the kernel of the dimension group representation discussed in [BK, BLR, W1, and W2]. Elements of $\operatorname{Aut}_{0}\left(\sigma_{A}\right)$ will be called inert. FOG asks more precisely whether $\operatorname{Aut}_{0}\left(\sigma_{A}\right)$ is generated by its finite order elements. We will apply (1.7), (1.16), and (1.22) to prove

Eventual FOG (1.1). Let $\alpha$ be in $\operatorname{Aut}_{0}\left(\sigma_{A}\right)$. There is an integer $k_{0} \geq 1$ such that if $k \geq k_{0}$, then $\alpha$ is a product of homeomorphisms of $X_{A}$ of finite order which commute with $\sigma_{A}^{k}$. Furthermore, each of these finite order elements is a simple element of $\operatorname{Aut}_{0}\left(\sigma_{A}^{k}\right)$.

In our proof of (1.1), the integer $k_{0}$ seems to depend on $\alpha$ and the product expressions are possibly different for different $k$. Of course, FOG conjectures that we can take $k_{0}=1$, in which case any product expression for $k=1$ works for all $k$. M. Boyle has recently refined (1.1) to show that each $\alpha \in \operatorname{Aut}_{0}\left(\sigma_{A}\right)$ is the product of two simple automorphisms in $\operatorname{Aut}_{0}\left(\sigma_{A}^{k}\right)$ for $k \geq n_{0}$ where, again, $n_{0}$ depends on $\alpha$ but is usually larger than $k_{0}$ (see [Boy]). If FOG turns out to be false in general, the challenge will then be to understand whether it does

Received by the editors June 21, 1988.

1980 Mathematics Subject Classification (1985 Revision). Primary 54H20, 57S99, 20F99; Secondary $60 \mathrm{~J} 10$.

Key words and phrases. Kernel of the dimension group representation, Eventual FOG, the space of strong shift equivalences, LIFT, $p$-adic asymptotic gyration numbers.

This research was partially supported by the NSF. 
hold in particular cases and to understand its relation to Eventual FOG. One of the main tools in the proof of Eventual FOG in the two-sided case is the space of strong shift equivalences $R S(\overline{\mathscr{E}})$ over the nonnegative integers. See (1.7) for its relation to Nasu's simple automorphisms, and see (1.8) and (1.9) for a brief discussion of how a strengthened version of the shift equivalence problem SHIFT is related to the problem of whether the dimension group representation is always surjective. The argument for Eventual FOG is outlined in this section. Detailed proofs are completed in $\S 2$. The reader who wishes to go on to some implications of FOG and Eventual FOG can skip immediately to $\S 3$ and $\S 4$. These sections are basically independent of the details in $\S 1$ and $\S 2$.

Thanks to B. Marcus for discussions on this material and, in particular, for his contribution to sharpening the results in $\S 3$. Thanks also to E. Thomas for his contribution to $\S 3$, to D. Bertrand for writing his Appendix I to [W] used in $\S 4$, and to the referee for helpful comments.

Incidentally, for one-sided subshifts of finite type the entire group of symmetries is indeed generated by elements of finite order (see [BFK]). The proof can be viewed as taking place in the context of a one-sided version of $R S(\overline{\mathscr{E}})$.

In addition to the space $R S(\overline{\mathscr{E}})$ of strong shift equivalences, we will use the space $S(\overline{\mathscr{E}})$ of shift equivalences introduced in [W1, W2] where $\mathscr{E}$ is the set of finite zero-one matrices and $\overline{\mathscr{E}}$ is the category of finite nonnegative integral matrices. In [W1] it was observed that there is an isomorphism

$$
\operatorname{Aut}\left(s_{A}\right) \approx \pi_{1}(S(\overline{\mathscr{E}}), A),
$$

where $\operatorname{Aut}\left(s_{A}\right)$ is the group of order preserving automorphisms of the dimension group $G(A)$ which commute with the automorphism $s_{A}$ induced on $G(A)$ by $A$. Also see [BLR] for recent information on $\operatorname{Aut}\left(s_{A}\right)$. In [W2] we constructed isomorphisms

$$
\begin{aligned}
& \Phi_{A}: \operatorname{Aut}\left(\sigma_{A}\right) \rightarrow \pi_{1}(R S(\mathscr{E}), A), \\
& \Theta_{A}: \pi_{1}(R S(\mathscr{E}), A) \rightarrow \operatorname{Aut}\left(\sigma_{A}\right)
\end{aligned}
$$

which are inverses of each other. FOG can therefore be reformulated to state

$$
\text { The kernel of } \delta_{A}: \pi_{1}(R S(\mathscr{E}), A) \rightarrow \pi_{1}(S(\overline{\mathscr{E}}), A)
$$

is generated by its elements of finite order.

We prove Eventual FOG by analyzing the homomorphism $\delta_{A}$.

As mentioned in [W1], the set of path components $\pi_{0}(R S(\mathscr{E}))$ is just the set of strong shift equivalence classes in $\mathscr{E}$ and $\pi_{0}(S(\overline{\mathscr{E}}))$ is the set of shift equivalence classes in $\overline{\mathscr{E}}$. Let $R S(\overline{\mathscr{E}})$ be the space of strong shift equivalences formed from the $R S$ Triangle Identities as in [W2] where now the matrices can be finite nonnegative integral and not just zero-one. Incidentally, we always assume that any matrix in $\mathscr{E}$ or $\overline{\mathscr{E}}$ has at least one nonzero entry in each row and each column. It is well known that strong shift equivalence in $\mathscr{E}$ is the same as strong shift equivalence in $\overline{\mathscr{E}}$. This translates literally into the 
statement that the natural map

$$
\pi_{0}(R S(\mathscr{E})) \rightarrow \pi_{0}(R S(\overline{\mathscr{E}}))
$$

is a bijection (see (2.10) below). If $(R, S): P \rightarrow Q$ in $R S(\overline{\mathscr{E}})$, then $R: P \rightarrow Q$ in $S(\overline{\mathscr{E}})$ and there is a sequence of canonical maps

$$
R S(\mathscr{E}) \rightarrow R S(\overline{\mathscr{E}}) \rightarrow S(\overline{\mathscr{E}})
$$

SHIFT translates into the question of whether

$$
\pi_{0}(R S(\overline{\mathscr{E}})) \rightarrow \pi_{0}(S(\overline{\mathscr{E}}))
$$

is a bijection. Of course, the mere reformulations (1.4) and (1.6) do not help to solve the problem. But they do have heuristic value, and a key point for us will be the difference between $R S(\mathscr{E})$ and $R S(\overline{\mathscr{E}})$ on the level of $\pi_{1}$.

Recall from $[\mathrm{N}]$ the definition of a simple element of $\operatorname{Aut}\left(\sigma_{A}\right)$. Let $\mathscr{S}$ be the set of edges of a finite directed graph. Define a zero-one matrix $P: \mathscr{S} \times \mathscr{S} \rightarrow$ $\{0,1\}$ by the condition that $P(x, y)=1$ if and only if the end point of the edge $x$ is the initial point of the edge $y$. Any automorphism of the graph which fixes the vertices induces a 1-block automorphism of $\left(X_{P}, \sigma_{P}\right)$. The finite subgroup of $\operatorname{Aut}\left(\sigma_{P}\right)$ generated by these symmetries will be denoted by $\Sigma_{P}$. Let $\sigma: X \rightarrow X$ be a homeomorphism. A simple symmetry in $\operatorname{Aut}(\sigma)$ is one of the form $\alpha \beta \alpha^{-1}$ where $\alpha:\left(X_{P}, \sigma_{P}\right) \rightarrow(X, \sigma)$ is a conjugacy between some subshift of finite type $\left(X_{P}, \sigma_{P}\right)$ and the dynamical system $(X, \sigma)$, and where $\beta \in \Sigma_{P}$. Under the isomorphism in (1.2), a simple element $\alpha$ in $\Sigma_{P}$ corresponds to the elementary strong shift equivalence $\left(\alpha^{-1}, \alpha P\right)$ in $\pi_{1}(R S(\mathscr{E}), P)$.

Theorem (1.7). Let $A$ be a square zero-one matrix. Then

$$
\Upsilon_{A}: \pi_{1}(R S(\mathscr{E}), A) \rightarrow \pi_{1}(R S(\overline{\mathscr{E}}), A)
$$

is surjective and the kernel is precisely the normal subgroup SSS generated by simple symmetries.

Consider the following part of the exact homotopy sequence associated to the map $R S(\overline{\mathscr{E}}) \rightarrow S(\overline{\mathscr{E}})$ :

$$
\begin{gathered}
\rightarrow \pi_{2}(S(\overline{\mathscr{E}}), R S(\overline{\mathscr{E}}) ; A) \rightarrow \pi_{1}(R S(\overline{\mathscr{E}}), A) \rightarrow \pi_{1}(S(\overline{\mathscr{E}}), A) \\
\rightarrow \pi_{1}(S(\overline{\mathscr{E}}), R S(\overline{\mathscr{E}}) ; A) \rightarrow \pi_{0}(R S(\overline{\mathscr{E}})) \rightarrow \pi_{0}(S(\overline{\mathscr{E}})) .
\end{gathered}
$$

The last map on the right is surjective. A stronger form of SHIFT is

Question (1.9). Does $\pi_{1}(S(\overline{\mathscr{E}}), R S(\overline{\mathscr{E}}) ; A)=0$ ?

Since $\pi_{1}(S(\overline{\mathscr{E}}), R S(\overline{\mathscr{E}}) ; A)$ is not a group, the question really asks whether every path from $A$ to $B$ in $S(\overline{\mathscr{E}})$ is homotopic in $S(\overline{\mathscr{E}})$ keeping end points fixed to a path in $R S(\overline{\mathscr{E}})$. 
Question (1.10). Does $\pi_{2}(S(\overline{\mathscr{E}}), R S(\overline{\mathscr{E}}) ; A)=0$ ?

In view of (1.7), an affirmative answer to this would imply a strong version of FOG; namely, that every element of the kernel $\operatorname{Aut}_{0}\left(\sigma_{A}\right)$ of the dimension group representation is a product of simple elements. On the other hand, it is easy to produce candidates for nonzero elements of the kernel of $\delta_{A}: \pi_{1}(R S(\overline{\mathscr{E}}), A) \rightarrow \pi_{1}(S(\overline{\mathscr{E}}), A)$. Consider two strong shift equivalences $(R, S): A \rightarrow A$ and $(R, T): A \rightarrow A$. Then the element

$$
\gamma(R, S) * \gamma(R, T)^{-1}
$$

of $\pi_{1}(R S(\overline{\mathscr{E}}), A)$ is in the kernel of $\delta_{A}$. In view of (1.7) it is then also relevant to ask

Question (1.12). Is every finite order element of $\operatorname{Aut}_{0}\left(\sigma_{A}\right)$ simple? ${ }^{1}$

Let $R S(\mathscr{E})_{A}$ be the component of $R S(\mathscr{E})$ to which $A$ belongs; similarly for $R S(\overline{\mathscr{E}})_{A}$ and $S(\overline{\mathscr{E}})_{A}$. The homology and homotopy groups of $R S(\mathscr{E})_{A}$ and $R S(\overline{\mathscr{E}})_{A}$ are invariants of strong shift equivalence by (1.4). In [W2] it was shown that $R S(\mathscr{E})_{A}$ is homotopy equivalent to the classifying space of the discrete group $\operatorname{Aut}\left(\sigma_{A}\right)$. So its homology is probably quite rich. For example, see the gyration number theory of $[B K],(7.8)$ of $[B L R]$, and [W1] for some information relevant to

$$
H_{1}\left(R S(\mathscr{E})_{A}\right)=H_{1}\left(\operatorname{Aut}\left(\sigma_{A}\right)\right)=\operatorname{Aut}\left(\sigma_{A}\right) /\left[\operatorname{Aut}\left(\sigma_{A}\right), \operatorname{Aut}\left(\sigma_{A}\right)\right] .
$$

A question related to $H_{2}\left(R S(\mathscr{E})_{A}\right)=H_{2}\left(\operatorname{Aut}\left(\sigma_{A}\right)\right)$ is whether $\operatorname{Aut}\left(\sigma_{A}\right)$ contains subgroups isomorphic to the fundamental group of a Riemann surface of genus $g$ for $g \geq 2$ whenever $A$ is aperiodic. These surface groups are known to be residually finite, which is a necessary condition for imbedding by (3.1) of [BLR].

The homology and homotopy groups of $S(\overline{\mathscr{E}})_{A}$ are invariants of shift equivalence. The result (1.16) below compares the homology and homotopy of $R S(\overline{\mathscr{E}})_{A}$ and $S(\overline{\mathscr{E}})_{A}$ under "eventualization". Any homeomorphism of $X_{A}$ which commutes with $\sigma_{A}$ also commutes with any of its powers $\sigma_{A}^{k}$. So $\operatorname{Aut}\left(\sigma_{A}\right)$ is a subgroup of $\operatorname{Aut}\left(\sigma_{A}^{k}\right)$. The analogue for $R S(\overline{\mathscr{E}})$ and $S(\overline{\mathscr{E}})$ goes as follows.

Let $(R, S): A \rightarrow B$ in $R S(\overline{\mathscr{E}})$ and let $k \geq 1$. Then $\left(R, S A^{k-1}\right): A^{k} \rightarrow B^{k}$ in $R S(\overline{\mathscr{E}})$. Moreover, if

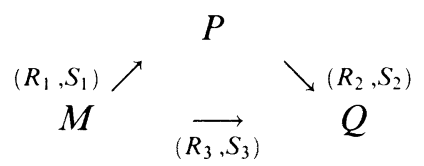

\footnotetext{
${ }^{1}$ Recent interesting examples given first by Kim-Roush and subsequently by Boyle-Fiebig show the answer to (1.10) and to (1.12) is "no".
} 
is a triangle in $R S(\overline{\mathscr{E}})$, then

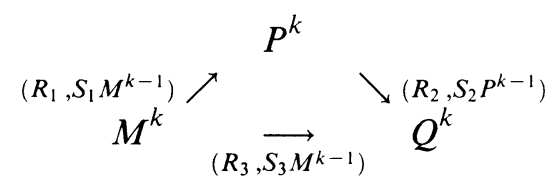

is also a triangle in $R S(\overline{\mathscr{E}})$. This gives a canonical "eventualization" map

$$
\mathrm{ev}_{k}: R S(\overline{\mathscr{E}})_{A} \rightarrow R S(\overline{\mathscr{E}})_{A^{k}} \text {. }
$$

Any shift equivalence $R: P \rightarrow Q$ is also a shift equivalence $R: P^{k} \rightarrow Q^{k}$. So we have the map

$$
\mathrm{ev}_{k}: S(\overline{\mathscr{E}})_{A} \rightarrow S(\overline{\mathscr{E}})_{A^{k}} .
$$

Moreover, there is a commutative diagram:

$$
\begin{aligned}
R S(\mathscr{E})_{A} \rightarrow R S(\overline{\mathscr{E}})_{A} & \rightarrow R S(\overline{\mathscr{E}})_{A^{k}} \\
\left.\frac{\downarrow}{(\mathscr{E})}\right)_{A} & \rightarrow S\left(\frac{1}{\mathscr{E}}\right)_{A^{k}}
\end{aligned}
$$

For simplicity we will let $\delta_{A}$ also denote the induced map on the homology or homotopy of $R S(\mathscr{E})_{A}$ and/or $R S(\overline{\mathscr{E}})_{A}$ to that of $S(\overline{\mathscr{E}})_{A}$. Similarly for $\mathrm{ev}_{k}$.

Theorem (1.16). (A) Let $\beta \in H_{n}\left(S(\overline{\mathscr{E}})_{A}\right)$. There is a $k_{0} \geq 1$ such that if $k \geq k_{0}$, then there is an element $\alpha \in H_{n}\left(R S(\overline{\mathscr{E}})_{A^{k}}\right)$ with $\delta_{A^{k}}(\alpha)=\mathrm{ev}_{k}(\beta)$.

(B) Suppose $\alpha$ in $H_{n}\left(R S(\mathscr{E})_{A}\right)$ or in $H_{n}\left(R S(\overline{\mathscr{E}})_{A}\right)$ satisfies $\delta_{A}(\alpha)=0$. There is a $k_{0} \geq 1$ such that if $k \geq k_{0}$, then $\operatorname{ev}_{k}(\alpha)=0$ in $H_{n}\left(R S\left(\frac{A}{\mathscr{E}}\right)_{A^{k}}\right)$.

(C) The same statements hold with $H_{n}$ replaced by $\pi_{n}$ throughout.

Using the surjectivity part of (1.7) and $\pi_{1}$ in (A) of (1.16) provides an alternate proof of (6.3) of [BLR]. As an example of (B) using $\pi_{1}$, consider the element (1.11) in the kernel of $\delta_{A}: \pi_{1}(R S(\overline{\mathscr{E}}), A) \rightarrow \pi_{1}(S(\overline{\mathscr{E}}), A)$. Since $S A=T A$, we have

$$
\mathrm{ev}_{2}\left(\gamma(R, S) * \gamma(R, T)^{-1}\right)=\gamma(R, S A) * \gamma(R, T A)^{-1}=1
$$

in $\pi_{1}(R S$ (overline $\left.\mathscr{E}), A^{2}\right)$. Under the isomorphism $\Theta_{A}$ of (1.2) the loop (1.11) corresponds to the self-conjugacy $c(R, S)^{-1} c(R, T)$ of $\left(X_{A}, \sigma_{A}\right)$. The proof of Eventual FOG will show that as a conjugacy of $\left(X_{A}, \sigma_{A^{2}}\right)$ this element is a product of simple elements.

Question (1.17). What is the relationship between $H_{n}\left(R S(\mathscr{E})_{A}\right)$ and $H_{n}\left(R S(\overline{\mathscr{E}})_{A}\right)$ ?

Now recall the standard "edge path" construction which associates a subshift of finite type to a nonnegative integral square matrix $A: X \times X \rightarrow \mathbf{Z}_{+}$. Let $A=\{A(i, j)\}$. Let $X^{\prime}=\{(i, \alpha, j) \mid 1 \leq \alpha \leq A(i, j)\}$ and define the zero-one matrix $A^{\prime}: X^{\prime} \times X^{\prime} \rightarrow\{0,1\}$ by the condition

$$
A^{\prime}\left(\left(i_{1}, \alpha_{1}, j_{1}\right),\left(i_{2}, \alpha_{2}, j_{2}\right)\right)=1 \quad \text { iff } j_{1}=i_{2} \text {. }
$$


Alternatively, we build a graph with vertices $i \in X$ and directed edges $x=$ $(i, \alpha, j)$ from $i$ to $j$. Then $A^{\prime}(x, y)=1$ iff the end point of $x$ is the initial point of $y$. The subshift $\left(X_{A^{\prime}}, \sigma_{A^{\prime}}\right)$ is the edge path presentation. Next define zero-one matrices $R_{A}: X \times X^{\prime} \rightarrow\{0,1\}$ and $S_{A}: X^{\prime} \times X \rightarrow\{0,1\}$ by the formulas

$$
\begin{array}{lll}
R_{A}\left(i,\left(i_{1}, \alpha_{1}, j_{1}\right)\right)=1 & \text { iff } & i=i_{1}, \\
S_{A}\left(\left(i_{1}, \alpha_{1}, j_{1}\right), j\right)=1 & \text { iff } & j_{1}=j .
\end{array}
$$

Direct computation shows

$$
A=R_{A} S_{A} \quad \text { and } \quad A^{\prime}=S_{A} R_{A} .
$$

Therefore we have $\left(R_{A}, S_{A}\right): A \rightarrow A^{\prime}$ in $R S(\overline{\mathscr{E}})$. Let $\gamma_{A}=\gamma\left(R_{A}, S_{A}\right)$ be the corresponding path in $R S(\overline{\mathscr{E}})$ from $A$ to $A^{\prime}$. The map sending a loop $\gamma$ in $\pi_{1}\left(R S(\overline{\mathscr{E}}), A^{\prime}\right)$ to the loop $\gamma *_{A} \gamma * \gamma_{A}^{-1}$ in $\pi_{1}(R S(\overline{\mathscr{E}}), A)$ gives an isomorphism

$$
\pi_{1}\left(R S(\overline{\mathscr{E}}), A^{\prime}\right) \rightarrow \pi_{1}(R S(\overline{\mathscr{E}}), A) \text {. }
$$

For any zero-one matrix $A$, let $\operatorname{Aut}\left(\sigma_{A}\right) \bmod \mathscr{S} \mathscr{S}$ denote $\operatorname{Aut}\left(\sigma_{A}\right)$ modulo the subgroup generated by the simple automorphisms; similarly for $\pi_{1}(R S(\mathscr{E}), A)$ mod $\mathscr{S} \mathscr{S}$. The final ingredient for proving Eventual FOG is to show there is a commutative diagram:

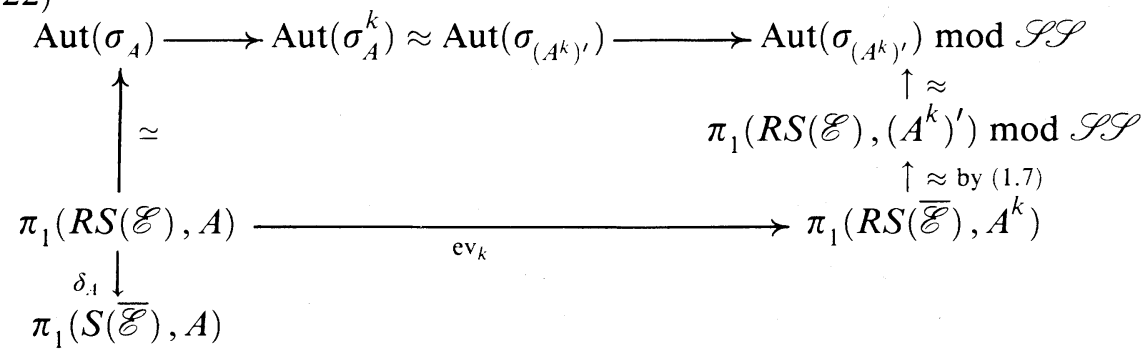

We can now easily complete the proof of Eventual FOG. Let $\alpha \in \operatorname{Aut}\left(\sigma_{A}\right)$ satisfy $\delta_{A} \Phi_{A}(\alpha)=1$. Then $\operatorname{ev}_{k}\left(\Phi_{A}(\alpha)\right)=1$ for $k \geq k_{0}$. Aut $\left(\sigma_{A}\right)$ is a subgroup of $\operatorname{Aut}\left(\sigma_{A}^{k}\right)$, and going round the top right side of (1.22) shows that $\alpha$ is a product of simple elements of finite order in $\operatorname{Aut}\left(\sigma_{A}^{k}\right)$.

Commutativity of (1.22) is more or less routine. The main points for understanding the preceding outline for (1.1) are in the proofs of (1.7) and (B) of (1.16) using $\pi_{1}$.

Throughout we adopt the following convention. Composition of maps between sets or spaces shall be read from right to left. If $\alpha: I \rightarrow K$ is a bijection of sets, we let $\alpha$ also denote the $K \times I$ matrix where $\alpha(k, i)=1$ iff $k=\alpha(i)$. If $A$ is an $I \times I$ matrix and $B$ is a $K \times K$ matrix such that $A(i, j)=B(\alpha(i), \alpha(j))$ for $(i, j) \in I \times I$, then we have $B=\alpha A \alpha^{-1}$.

\section{StRong SHIfT EQUiVALENCE OVER THE NONNEGATIVE INTEGERS}

Proof of (1.7). First we show that the simple elements $\left(\alpha^{-1}, \alpha P\right)$ of $\pi_{1}(R S(\mathscr{E}), A)$ lie in the kernel of $\Upsilon_{A}$. Let $\mathscr{T}$ be the set of vertices of the 
graph defining $P$, and let $Q$ be the $\mathscr{T} \times \mathscr{T}$ matrix in $\overline{\mathscr{E}}$ given by

$$
Q\left(t, t^{\prime}\right)=\text { number of directed edges in the graph from } t \text { to } t^{\prime} \text {. }
$$

Define the $\mathscr{T} \times \mathscr{S}$ matrix $R$ and the $\mathscr{S} \times \mathscr{T}$ matrix $S$ by the conditions

$$
\begin{aligned}
& R(t, s)=1 \text { if and only if } t \text { is the initial point of } s, \\
& S(s, t)=1 \text { if and only if } t \text { is the final point of } s .
\end{aligned}
$$

We then have the triangle

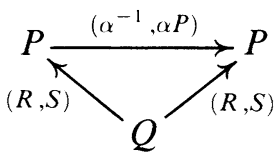

in $R S(\overline{\mathscr{E}})$, and this shows the loop $\gamma\left(\alpha^{-1}, \alpha P\right)$ is homotopic to a constant.

Next consider $(R, S): P \rightarrow Q$ in $R S(\overline{\mathscr{E}})$ where $P: X \times X \rightarrow \mathbf{Z}_{+}$and $Q: Y \times$ $Y \rightarrow \mathbf{Z}_{+}$. We shall produce a commutative diagram

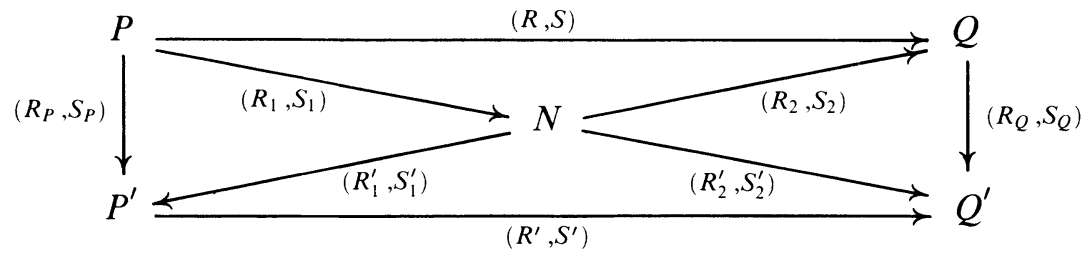

in $R S(\overline{\mathscr{E}})$ where $P^{\prime}: X^{\prime} \times X^{\prime} \rightarrow\{0,1\},\left(R_{P}, S_{P}\right): P \rightarrow P^{\prime}, Q^{\prime}: Y^{\prime} \times Y^{\prime} \rightarrow$ $\{0,1\},\left(R_{Q}, S_{Q}\right): Q \rightarrow Q^{\prime}$ are as in (1.18), (1.19), and (1.20). Fix a pair of indices $(i, j)$ in $X \times X$. The equation

$$
P(i, j)=\sum_{k} R(i, k) S(k, j)
$$

implies there is a bijection between the set

$$
\{(i, \alpha, j) \mid 1 \leq \alpha \leq P(i, j)\}
$$

and the set

$$
\{((i, \beta, k),(k, \gamma, j)) \mid 1 \leq \beta \leq R(i, k) \text { and } 1 \leq \gamma \leq S(k, j)\} .
$$

Using graph terminology, this means there is a bijection between the (directed) edges $x=(i, \alpha, j)$ from $i$ to $j$ determined by $P$ and pairs of edges $y z$ where $y=(i, \beta, k)$ is an edge from $i$ to $k$ determined by $R$ and $z=(k, \gamma, j)$ is an edge from $k$ to $j$ determined by $S$. We will call $y$ the tail and $z$ the head. In particular, the initial point of $y$ is $i$ and the final point of $y$ is $k$. The initial point of $z$ is $k$ and the final point of $z$ is $j$. A choice of bijection

$$
p(x)=y z
$$

from (2.2) to (2.3) will be denoted by $p: P \approx R S$. 
Define matrices $R^{\prime}: X^{\prime} \times Y^{\prime} \rightarrow\{0,1\}$ and $S^{\prime}: Y^{\prime} \times X^{\prime} \rightarrow\{0,1\}$ as follows: Make a choice of bijections

$$
p: P \approx R S \text { and } q: Q \approx S R .
$$

Let $x=(i, \alpha, j)$ be in $X^{\prime}$ and $y=(k, \beta, l)$ be in $Y^{\prime}$. Then set

$$
\begin{array}{lll}
R^{\prime}(x, y)=1 & \text { iff } & \text { head of } p(x)=\text { tail of } q(y), \\
S^{\prime}(y, x)=1 & \text { iff } & \text { head of } q(y)=\text { tail of } p(x) .
\end{array}
$$

We have

$$
P^{\prime}=R^{\prime} S^{\prime} \quad \text { and } \quad Q^{\prime}=S^{\prime} R^{\prime}
$$

Let $Z=\{(i, \alpha, k) \mid 1 \leq \alpha \leq R(i, k)\}$ and define the matrix $N: Z \times Z \rightarrow \mathbf{Z}_{+}$by the equation

$$
N((i, \alpha, k),(j, \beta, l))=S(k, j) .
$$

Define the other matrices in (2.1) to be

$$
\begin{aligned}
& R_{1}(i,(j, \alpha, k))=1 \quad \text { iff } i=j, \\
& S_{1}((j, \alpha, k), i)=S(k, i), \\
& R_{2}((i, \alpha, k), l)=1 \quad \text { iff } \quad k=l, \\
& S_{2}(l,(i, \alpha, k))=S(l, i), \\
& R_{1}^{\prime}\left((i, \alpha, k),\left(i_{1}, \beta, i_{2}\right)\right)=1 \quad \text { iff } \quad(i, \alpha, k)=\text { tail of } p\left(\left(i_{1}, \beta, i_{2}\right)\right), \\
& S_{1}^{\prime}\left(\left(i_{1}, \beta, i_{2}\right),(i, \alpha, k)\right)=1 \quad \text { iff } i_{2}=i, \\
& R_{2}^{\prime}((i, \alpha, k),(l, \beta, m))=1 \text { iff } k=l, \\
& S_{2}^{\prime}((l, \beta, m),(i, \alpha, k))=1 \quad \text { iff head of } q((l, \beta, m))=(i, \alpha, k) .
\end{aligned}
$$

Verification that these definitions give the commutative diagram (2.1) is straightforward.

Corollary (2.10). $\pi_{0}(R S(\mathscr{E})) \rightarrow \pi_{0}(R S(\overline{\mathscr{E}}))$ is a bijection.

This map is surjective by (1.20) and injective by (2.1).

Let $\gamma=\gamma(R, S) \in \pi_{1}(R S(\overline{\mathscr{E}}) ; P, Q)$ be the homotopy class of paths from $P$ to $Q$ determined by $(R, S): P \rightarrow Q$. Similarly for $\gamma^{\prime}=\gamma\left(R^{\prime}, S^{\prime}\right)$ in $\pi_{1}\left(R S(\mathscr{E}) ; P^{\prime}, Q^{\prime}\right)$. The path $\gamma^{\prime}$ depends on the choice made in (2.5). However, the key point for proving (1.7) is

Lemma (2.11). Another choice of bijections $p$ and $q$ in (2.5) changes $\gamma^{\prime}$ to a path of the form $\alpha * \gamma^{\prime} * \beta$ where $\alpha \in \pi_{1}\left(R S(\mathscr{E}), P^{\prime}\right)$ and $\beta \in \pi_{1}\left(R S(\mathscr{E}), Q^{\prime}\right)$ are simple elements of finite order.

Proof. We give the argument when $p$ changes but $q$ does not. The other case when $q$ alone changes is virtually the same. The new $p$ will be of the form $p a$ where $a: X^{\prime} \rightarrow X^{\prime}$ is a bijection which for each pair of indices $(i, j) \in X \times X$ takes the set (2.2) to itself. Viewing $a$ as a permutation matrix, 
we have $a P^{\prime}=P^{\prime} a=P^{\prime}$. Let $R$ and $S$ denote the matrices coming from $p a$ and from $q=q$ as in (2.6). Then $R=a R^{\prime}$ and $S=S^{\prime} a^{-1}$. The triangle

$$
\stackrel{\left(a^{-1}, P^{\prime}\right) \nearrow}{P^{\prime}} \underset{\left(\overrightarrow{R^{\prime}, S^{\prime}}\right)}{\longrightarrow} P^{\prime} Q^{\prime}
$$

in $R S(\mathscr{E})$ shows that $\gamma^{\prime}=\alpha * \gamma$ where $\gamma=\gamma(R, S)$ and $\alpha=\gamma\left(a^{-1}, P^{\prime}\right)$. It is easy to verify that $\alpha^{k}=\gamma\left(a^{-k}, P^{\prime}\right)$ for all $k \geq 1$. Since $X^{\prime}$ is a finite set, we have $a^{-n}=1$ for some $n$ and therefore $\alpha^{n}=1$ by 3.3(a) of [W2].

Remark (2.12). From (2.11) we have

$$
\alpha * \gamma^{\prime} * \beta=\gamma^{\prime} *\left[\left(\left(\gamma^{\prime}\right)^{-1} * \alpha *\left(\gamma^{\prime}\right)\right) * \beta\right]=\left[\alpha *\left(\left(\gamma^{\prime}\right) * \beta *\left(\gamma^{\prime}\right)^{-1}\right)\right] * \gamma^{\prime} .
$$

This says that changing (2.5) only changes $\gamma^{\prime}$ by products of simple symmetries of $P^{\prime}$ on the left or by products of simple symmetries of $Q^{\prime}$ on the right.

Surjectivity of $\Upsilon_{A}$. Any loop $\gamma \in \pi_{1}(R S(\overline{\mathscr{E}}), A)$ is a product of elementary edges $\gamma(R, S)^{\varepsilon}$ for $\varepsilon= \pm 1$. Make a choice (2.5) for each edge in the loop. Then we have the corresponding loop $\gamma^{\prime} \in \pi_{1}\left(R S(\mathscr{E}), A^{\prime}\right)$ which is the product of the various $\gamma\left(R^{\prime}, S^{\prime}\right)^{\varepsilon}$. Putting the diagrams (2.1) together forms a homotopy between $\gamma$ and $\gamma_{A} * \gamma^{\prime} * \gamma_{A}^{-1} \in \pi_{1}(R S(\mathscr{E}), A)$. In particular, a loop

$$
\gamma=\prod_{i} \gamma\left(R_{i}, S_{i}\right)^{\varepsilon_{i}}
$$

in $\pi_{1}(R S(\overline{\mathscr{E}}), A)$ gives rise to a loop

$$
\gamma^{\prime}=\prod_{i} \gamma\left(R_{i}^{\prime}, S_{i}^{\prime}\right)^{\varepsilon_{i}}
$$

which is well defined in $\pi_{1}\left(R S(\mathscr{E}), A^{\prime}\right)$ modulo $\mathscr{S} \mathscr{S}$.

Finite order generation for the kernel of $\Upsilon_{A}$. It suffices to show there is a commutative diagram of homomorphisms

$$
\begin{gathered}
\pi_{1}(R S(\mathscr{E}), A) \longrightarrow \pi_{1}(R S(\mathscr{E}), A) \bmod \mathscr{S S} \\
\Upsilon_{A} \searrow \\
\pi_{1}(R S(\overline{\mathscr{E}}), A)
\end{gathered}
$$

where $\Omega_{A}(\gamma)=\gamma *_{A} \gamma^{\prime} * \gamma_{A}^{-1}$.

If $(R, S) ; P \rightarrow Q$ in $R S(\mathscr{E})$, then all the matrices in (2.1) are zero-one. If $\gamma \in \pi_{1}(R S(\mathscr{E}), A)$, the homotopy between $\gamma$ and $\gamma *{ }_{A} \gamma^{\prime} * \gamma_{A}^{-1}$ therefore lies in $R S(\mathscr{E})$. This shows $(2.14)$ is commutative once it is verified that $\Omega_{A}$ is well defined modulo $\mathscr{S} \mathscr{S}$. We know from (2.11) and (2.12) that this holds whenever the choices (2.5) change. It remains to show it also holds whenever $\gamma$ is deformed by a homotopy in $R S(\overline{\mathscr{E}})$. This follows immediately from $(2.17)$ 
below. In $R S(\overline{\mathscr{E}})$ consider the triangle:

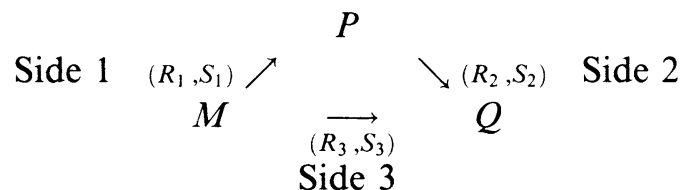

For each side make a choice of bijections as in (2.5) to produce a diagram of three edges in $R S(\mathscr{E})$ :

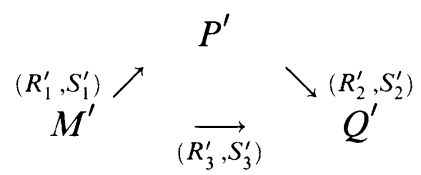

This is not necessarily a triangle in $R S(\mathscr{E})$.

Lemma (2.17). Given a choice of bijection (2.5) for Side 3, it is possible to choose bijections (2.5) for Side 1 and for Side 2 so that (2.16) becomes a triangle in $R S(\mathscr{E})$.

Proof. Let $e: M \approx R_{3} S_{3}$ and $f: Q \approx S_{3} R_{3}$ be as in (2.5). Using the $R S$ Triangle Identities for (2.15), we can also choose bijections $r: R_{3} \approx R_{1} R_{2}$, $s: S_{1} \approx R_{2} S_{3}$, and $t: S_{2} \approx S_{3} R_{1}$.

Step 1. The bijection $a: M \approx R_{1} S_{1}$. Let $x$ be an edge in (the edge presentation for) $M^{\prime}$ from the vertex $i$ to the vertex $j$. Write $e(x)=x_{1} x_{2}$ where the tail $x_{1}$ is an edge in $R_{3}^{\prime}$ and the head $x_{2}$ is in $S_{3}^{\prime}$. Now let $r\left(x_{1}\right)=y_{1} y_{2}$ where $y_{1}$ is the tail in $R_{1}^{\prime}$ and $y_{2}$ is the head in $R_{2}^{\prime}$. Finally, we let $a(x)=y_{1} z$ where $z$ is the unique edge in $S_{1}^{\prime}$ such that $s(z)=y_{2} x_{2}$. In terms of the graph presentation, this looks like:

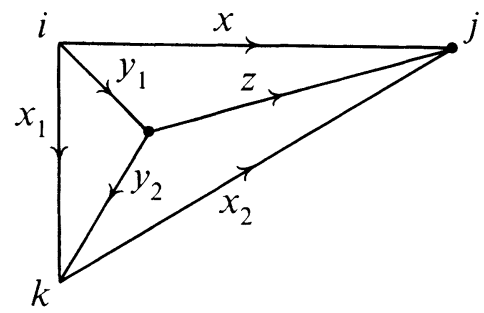

To prove this gives a bijection $a: M \approx R_{1} S_{1}$, we first show the correspondence sending $x$ to $y_{1} z$ is injective. Let $\bar{x}$ be another edge from $i$ to $j$ in $M^{\prime}$, and let $e(\bar{x})=\bar{x}_{1} \bar{x}_{2}$. Since $x \neq \bar{x}$, either $x_{1} \neq \bar{x}_{1}$ or $x_{2} \neq \bar{x}_{2}$.

Case 1. $x_{1} \neq \bar{x}_{1}$. Let $r\left(\bar{x}_{1}\right)=\bar{y}_{1} \bar{y}_{2}$. If $y_{1} \neq \bar{y}_{1}$, then $y_{1} z \neq \bar{y}_{1} z$ and $a(x) \neq$ $a(\bar{x})$. So assume $y_{1}=\bar{y}_{1}$; then $y_{2} \neq \bar{y}_{2}$. But this implies $z \neq \bar{z}$ and hence $a(x) \neq a(\bar{x})$.

Case 2. $x_{2} \neq \bar{x}_{2}$. Then $z \neq \bar{z}$, because $s(z)=y_{2} x_{2}$ and $s(\bar{z})=\bar{y}_{2} \bar{x}_{2}$. Thus $a(x) \neq a(\bar{x})$.

Next we show surjectivity. Let $y_{1} z$ be the concatenation of the edge $y_{1}$ from $i$ to $k$ in $R_{1}^{\prime}$ followed by an edge $z$ from $k$ to $j$ in $S_{1}^{\prime}$. Let $s(z)=y_{2} x_{2}$, 
and then let $x_{1}$ be the edge in $R_{3}^{\prime}$ so that $r\left(x_{1}\right)=y_{1} y_{2}$. Finally, let $x$ be the edge in $M^{\prime}$ such that $e(x)=x_{1} x_{2}$. Then $a(x)=y_{1} z$.

Step 2. Construction of the bijection $h: S_{1} R_{1} \approx R_{2} S_{2}$. Let $z w$ be an edge in $S_{1}^{\prime} R_{1}^{\prime}$ consisting of a tail $z$ in $S_{1}^{\prime}$ and a head $w$ in $R_{1}^{\prime}$. Write $s(z)=y x$ where the tail $y$ is in $R_{2}^{\prime}$ an the head $x$ is in $S_{3}^{\prime}$. Next let $v$ be the unique edge in $S_{2}^{\prime}$ such that $t(v)=x w$. Finally, we let $h(z w)=y v$. The proof that this is a bijection as desired is straightforward. Compare Step 1.

Step 3. Choose a bijection $b: P \approx S_{1} R_{1}$.

Step 4. Let $c: P \approx R_{2} S_{2}$ be defined as $c=h b$.

Step 5. The bijection $d: Q \approx S_{2} R_{2}$. This is similar to Step 1. Let $x$ be an edge in $Q^{\prime}$. Write $f(x)=x_{1} x_{2}$ where the tail $x_{1}$ is an edge in $S_{3}^{\prime}$ and the head $x_{2}$ is in $R_{3}^{\prime}$. Now let $r\left(x_{2}\right)=y_{1} y_{2}$ where $y_{1}$ is the tail $R_{1}^{\prime}$ and $y_{2}$ is the head in $R_{2}^{\prime}$. Finally, we let $a(x)=z y_{2}$ where $z$ is the unique edge in $S_{2}^{\prime}$ such that $s(z)=x_{1} y_{1}$.

Now we let $\left(R_{1}^{\prime}, S_{1}^{\prime}\right): M^{\prime} \rightarrow P^{\prime}$ be defined using $a: M \approx R_{1} S_{1}$ and $b: P \approx$ $S_{1} R_{1}$, and let $\left(R_{2}^{\prime}, S_{2}^{\prime}\right): P^{\prime} \rightarrow Q^{\prime}$ be defined using $c: P \approx R_{2} S_{2}$ and $d: Q \approx$ $S_{2} R_{2}$. Again, the proof that (2.16) is a triangle in $R S(\mathscr{E})$ is straightforward using the definitions in Step 1 through Step 5.

This completes the argument for (1.7).

Proof of (1.16). We will prove (A) of (1.16) for homology $H_{n}$ and (B) of (1.16) for $\pi_{1}$. The argument in the order cases is similar.

Consider finite square matrices $P$ and $Q$ over $\mathbf{Z}_{+}$, and let $E$ be a finite matrix over $\mathbf{Z}_{+}$satisfying $P E=E Q$. Then $E$ induces a homomorphism of dimension groups $g(E): G(P) \rightarrow G(Q)$. Suppose $F$ is also a matrix over $\mathbf{Z}_{+}$ satisfying $P F=F Q$.

Lemma (2.18). $g(E)=g(F)$ if and only if $E Q^{m}=F Q^{m}$ for all integers $m$ greater than or equal to some positive integer $m_{0}$.

This is a well-known property of homomorphisms between direct limits of matrices of finite size.

Remember that $R: P \rightarrow Q$ in $S(\mathscr{E})$ means there is a matrix $S$ over $\mathrm{Z}_{+}$ and an $m_{0} \geq 1$ satisfying the shift equivalence conditions

$$
P R=R Q, \quad R S=P^{m}, \quad Q S=S P, \quad S R=Q^{m}
$$

whenever $m \geq m_{0}$. Consider a triangle

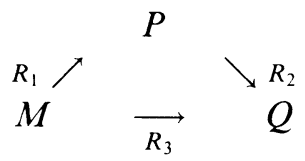

in $S(\overline{\mathscr{E}})$. We have $R_{1} R_{2}=R_{3}$. For each edge $R: P \rightarrow Q$ in (2.20), choose an $S$ and choose an $m \geq 1$ so that (2.19) holds and thus $(R, S): P^{m} \rightarrow Q^{m}$ in 
$R S(\overline{\mathscr{E}})$. Since there are only finitely many edges in the triangle (namely, three) we can choose all the $m$ 's to be the same. This gives a diagram

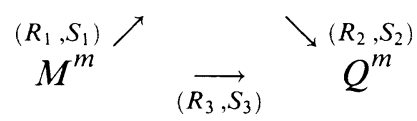

which is not necessarily a triangle in $R S(\overline{\mathscr{E}})$, because the last two equations of the $R S$ Triangles Identities involving the $S$-matrices may not (yet) hold. We claim there is an integer $h$ such that for $k \geq h$ the diagram

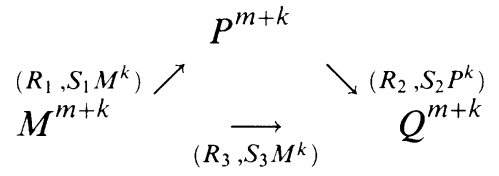

is a triangle in $R S(\overline{\mathscr{E}})$. The first equation $R_{1} R_{2}=R_{3}$ has not been altered. It remains to show there is an $h$ with

$$
S_{1} M^{k}=R_{2}\left(S_{3} M^{k}\right)=\left(R_{2} S_{3}\right) M^{k} \quad \text { and } \quad S_{2} P^{k}=\left(S_{3} M^{k}\right) R_{1}=\left(S_{3} R_{1}\right) P^{k}
$$

for $k \geq h$. By (2.18) and (2.19) it suffices to show

$$
g\left(S_{1}\right)=g\left(R_{2}\right) g\left(S_{3}\right)=g\left(R_{2} S_{3}\right) \text { and } g\left(S_{2}\right)=g\left(S_{3}\right) g\left(R_{1}\right)=g\left(S_{3} R_{1}\right) .
$$

Each edge in (2.21) is of the form $(R, S): E^{n} \rightarrow F^{n}$ where (2.19) also holds. Therefore, $g(R) g(S)=g(R S)=g\left(E^{n}\right)=g(E)^{n}$ and $g(S)=g(R)^{-1} g(E)^{n}$. We then compute

$$
g\left(R_{2}\right) g\left(S_{3}\right)=g\left(R_{2}\right) g\left(R_{3}\right)^{-1} g(M)^{n}=g\left(R_{1}\right)^{-1} g(M)^{n}=g\left(S_{1}\right)
$$

and

$$
\begin{aligned}
g\left(S_{3}\right) g\left(R_{1}\right) & =g\left(R_{3}\right)^{-1} g(M)^{n} g\left(R_{1}\right)=g\left(R_{3}\right)^{-1} g\left(R_{1}\right) g(P)^{n} \\
& =g\left(R_{2}\right)^{-1} g(P)^{n}=g\left(S_{2}\right) .
\end{aligned}
$$

Proof of $(\mathrm{A})$. An element $\beta$ in $H_{n}(S(\overline{\mathscr{E}}))$ is represented by a finite integral linear combination $\beta=\sum n_{r} S_{r}$ where we recall from [W1] that each $S_{r}$ is an $n$-simplex in $S(\overline{\mathscr{E}})$ consisting of an $(n+1)$-tuple $\left\langle A_{0}, \ldots, A_{n}\right\rangle$ together with shift equivalences $R_{i j}: A_{i} \mapsto A_{j}$ for $i<j$ satisfying $R_{i j} R_{j k}=R_{i k}$. Consider the collection of edges in $S(\overline{\mathscr{E}})$ which belong to at least one of the simplices $S_{r}$. For each such edge $R: P \rightarrow Q$, choose an $S$ so that $(R, S): P^{m} \rightarrow Q^{m}$ for some $m$. Since there are only finitely many edges, we can choose all the $m$ 's to be the same by (2.19). For any subtriangle of the form (2.20) lying in an $S_{r}$, there is the corresponding diagram (2.21). Again, since there are only finitely many simplices, we can choose an $h$ so that all the resulting diagrams (2.22) are triangles in $R S(\overline{\mathscr{E}})$. The required $\alpha$ in $H_{n}\left(R S(\overline{\mathscr{E}})_{A^{m+h}}\right)$ which maps to $\mathrm{ev}_{m+h}(\beta)$ under $\delta_{A^{m+h}}$ is the sum $\alpha=\sum n_{r} T_{r}$ where $T_{r}$ is obtained from $S_{r}$ by replacing each vertex $P$ of $S_{r}$ by $P^{m+h}$ and each edge $R: P \rightarrow Q$ of $S_{r}$ by the edge $\left(R, S P^{h}\right): P^{m+h} \rightarrow Q^{m+h}$. 
Proof of (B) for $\pi_{1}$. Consider a loop $\alpha \in \pi_{1}(R S(\overline{\mathscr{E}}), A)$ lying in the kernel of $\delta_{A}$. Represent $\alpha$ as a product of various $\gamma(R, S)^{\varepsilon}$ where $\varepsilon= \pm 1$ and $(R, S): P \rightarrow Q$. The image $\beta=\delta_{A}(\alpha)$ in $\pi_{1}(S(\overline{\mathscr{E}}), A)$ is represented by the product of the corresponding $\gamma(R)^{\varepsilon}$ where $R: P \rightarrow Q$.

Since we are assuming that $\beta=1$, it is possible to span this loop by a 2-dimensional disc in $S(\overline{\mathscr{E}})$ which is triangulated by 2-simplices of the form (2.20). For each edge $R: P \rightarrow Q$ in the interior of the 2-disc, choose an $S$ and choose an $n \geq 1$ so that (2.19) holds and thus $(R, S): P^{n} \rightarrow Q^{n}$. There are only finitely many edges in the 2-disc. Therefore, replacing each $S$ and $n$ by $S P^{k}$ and $n+k$ for large enough $k$ if necessary, we can assume (2.19) holds and $(R, S): P^{n} \rightarrow Q^{n}$ using the same $n=m$ for all the edges in the interior. Apply the eventualization map $\mathrm{ev}_{m}$ to the edges $(R, S): P \rightarrow Q$ in the loop $\alpha$ to get $\left(R, S P^{m-1}\right): P^{m} \rightarrow Q^{m}$ corresponding to the edges around the boundary of the 2-disc. For each triangle (2.20) in the 2-disc we have at this stage produced a diagram as in (2.21) which is not necessarily a triangle in $R S(\mathscr{E})$. If we knew these diagrams were indeed triangles in $R S(\mathscr{E})$, then they would make up a 2-disc spanning $\mathrm{ev}_{m}(\alpha)$. So to complete the proof choose a large $h$ so that (2.22) holds for each triangle. Then these triangles form a 2-disc spanning $\mathrm{ev}_{m+h}(\alpha)$ in $R S(\overline{\mathscr{E}})_{A^{m+h}}$.

Proof that (1.22) is commutative. Loops representing elements in the group $\pi_{1}(R S(\mathscr{E}), A)$ are products of $\gamma(R, S) \in \pi_{1}(R S(\mathscr{E}) ; P, Q)$ or their inverses coming from elementary strong shift equivalences $(R, S): P \rightarrow Q$. We will verify that the diagram (2.25) below is commutative when evaluated on $\gamma(R, S)$. Commutativity of (1.22) then follows, because the maps in (2.25) are all compatible with the products

$$
\operatorname{Isom}\left(\sigma_{N}, \sigma_{P}\right) \times \operatorname{Isom}\left(\sigma_{P}, \sigma_{Q}\right) \rightarrow \operatorname{Isom}\left(\sigma_{N}, \sigma_{Q}\right)
$$

and

$$
\pi_{1}(R S(\mathscr{E}) ; N, P) \times \pi_{1}(R S(\mathscr{E}) ; P, Q) \rightarrow \pi_{1}(R S(\mathscr{E}) ; N, Q) .
$$

Let $M$ and $N$ be square zero-one matrices. $\mathscr{S S}_{M}$ and $\mathscr{S S}_{N}$ denote respectively the subgroups generated by the simple symmetries in $\operatorname{Aut}\left(\sigma_{M}\right)$ and $\operatorname{Aut}\left(\sigma_{N}\right)$. The group $\mathscr{S S}_{M} \times \mathscr{S S}_{N}$ acts on the set $\operatorname{Isom}\left(\sigma_{M}, \sigma_{N}\right)$ by the formula $(\alpha, \beta) \cdot \gamma=\beta \gamma \alpha^{-1}$, and we let $\operatorname{Isom}\left(\sigma_{M}, \sigma_{N}\right) \bmod \mathscr{S} \mathscr{S}$ denote the equivalence classes of this action; similarly for $\pi_{1}(R S(\mathscr{E}) ; M, N) \bmod \mathscr{S} \mathscr{S}$. Let $M: \mathscr{S} \times \mathscr{S} \rightarrow\{0,1\}$ be a zero-one matrix and fix an integer $n \geq 1$. Let $\mathscr{S}(n)$ denote the union over all pairs $(i, j) \in \mathscr{S} \times \mathscr{S}$ of those sets

$$
\left\{\left(i_{0}, \ldots, i_{n}\right) \mid i_{0}=i, i_{n}=j \text {, and } M\left(i_{0}, i_{1}\right) M\left(i_{1}, i_{2}\right) \cdots M\left(i_{n-1}, i_{n}\right)=1\right\}
$$

which are nonempty. Let $M(n): \mathscr{S}(n) \times \mathscr{S}(n) \rightarrow\{0,1\}$ be the zero-one matrix defined by

$$
M(n)\left(\left(i_{0}, \ldots, i_{n}\right),\left(j_{0}, \ldots, j_{n}\right)\right)=1 \quad \text { iff } \quad i_{n}=j_{0} .
$$


The main diagram is

(2.25)

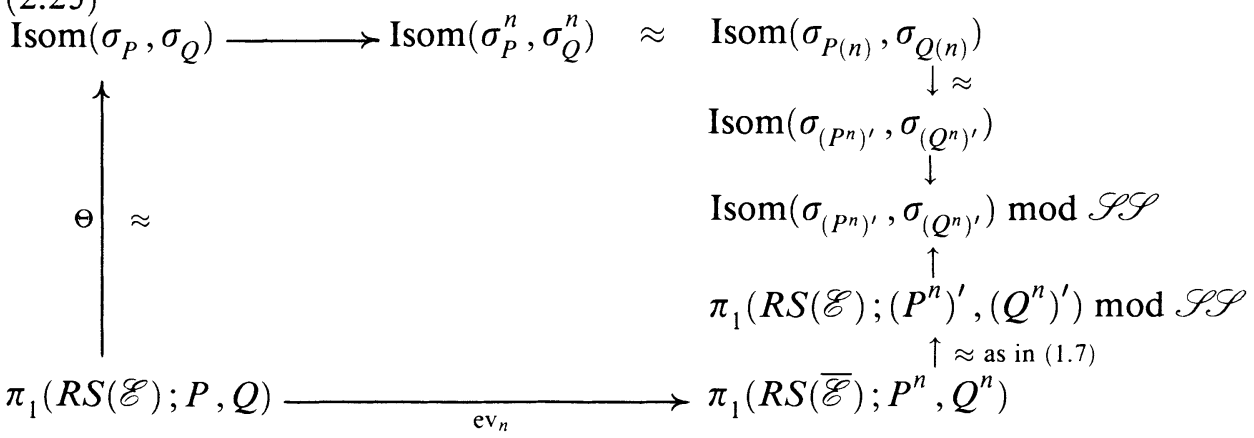

For any square zero-one matrix $M$, let $\alpha_{M}: X_{M} \rightarrow X_{M(n)}$ be the homeomorphism sending $x=\left\{x_{k}\right\}$ to $y=\left\{y_{k}\right\}$ where $y_{k}=\left(x_{n k}, x_{n k+1}, \ldots, x_{n(k+1)}\right)$. We have $\alpha_{M} \sigma_{M}^{n}=\sigma_{M(n)} \alpha_{M}$, and the bijection

$$
\operatorname{Isom}\left(\sigma_{P}^{n}, \sigma_{Q}^{n}\right) \approx \operatorname{Isom}\left(\sigma_{P(n)}, \sigma_{Q(n)}\right)
$$

sends $\gamma \in \operatorname{Isom}\left(\sigma_{P}^{n}, \sigma_{Q}^{n}\right)$ to $\alpha_{Q} \gamma \alpha_{P}^{-1} \in \operatorname{Isom}\left(\sigma_{P(n)}, \sigma_{Q(n)}\right)$. Define zero-one matrices $R(n)$ and $S(n)$ by the conditions

$$
\begin{aligned}
& R(n)\left(\left(i_{0}, \ldots, i_{n}\right),\left(k_{0}, \ldots, k_{n}\right)\right)=1 \\
& \quad \text { iff } R\left(i_{0}, k_{0}\right) S\left(k_{0}, i_{1}\right) R\left(i_{1}, k_{1}\right) S\left(k_{1}, i_{2}\right) \cdots S\left(k_{n-1}, i_{n}\right) R\left(i_{n}, k_{n}\right)=1, \\
& S(n)\left(\left(k_{0}, \ldots, k_{n}\right),\left(i_{0}, \ldots, i_{n}\right)\right)=1 \quad \text { iff } S\left(k_{n-1}, i_{0}\right) R\left(i_{0}, k_{n}\right) S\left(k_{n}, i_{1}\right)=1 .
\end{aligned}
$$

Then $P(n)=R(n) S(n) \quad Q(n)=S(n) R(n)$ and the bijection (2.26) takes $c(R, S): X_{P} \rightarrow X_{Q}$ to $c(R(n), S(n)): X_{P(n)} \rightarrow X_{Q(n)}$.

Let $P: \mathscr{S} \times \mathscr{S} \rightarrow\{0,1\}$ be a zero-one matrix and fix $n \geq 1$. For each pair of indices $(i, j)$ in $\mathscr{S} \times \mathscr{S}$, choose a bijection from the set $(2.24)$ for the matrix $P$ to the set $(2.2)$ for the matrix $P^{n}$. This gives a bijection $\beta_{P}$ from the set of states for $P(n)$ to the set of states for $\left(P^{n}\right)^{\prime}$ which we denote by

$$
\beta_{P}: P(n) \approx\left(P^{n}\right)^{\prime}
$$

Moreover, we have $\left(P^{n}\right)^{\prime}=\beta_{P} P(n) \beta_{P}^{-1}$, and $\beta_{P}$ induces a one-block conjugacy $\beta_{P}:\left(X_{P(n)}, \sigma_{P(n)}\right) \rightarrow\left(X_{\left(P^{n}\right)^{\prime}}, \sigma_{\left(P^{n}\right)^{\prime}}\right)$. If $\gamma_{P}: P(n) \approx\left(P^{n}\right)^{\prime}$ is another choice as in (2.28), then the compositions of conjugacies $\beta_{P}^{-1} \gamma_{P}$ and $\beta_{P} \gamma_{P}^{-1}$ lie respectively in the finite groups $\Sigma_{P(n)}$ and $\Sigma_{\left(p^{n}\right)^{\prime}}$. Let $Q: \mathscr{T} \times \mathscr{T} \rightarrow\{0,1\}$ be another square zero-one matrix and choose $\beta_{Q}: Q(n) \approx\left(Q^{n}\right)^{\prime}$ as in (2.28). This leads to a bijection

$$
\operatorname{Isom}\left(\sigma_{P(n)}, \sigma_{Q(n)}\right) \approx \operatorname{Isom}\left(\sigma_{\left(P^{n}\right)^{\prime}}, \sigma_{\left(Q^{n}\right)^{\prime}}\right)
$$


sending $\gamma \in \operatorname{Isom}\left(\sigma_{P(n)}, \sigma_{Q(n)}\right)$ to $\beta_{Q} \gamma \beta_{P}^{-1} \in \operatorname{Isom}\left(\sigma_{\left(P^{n}\right)^{\prime}}, \sigma_{\left(Q^{n}\right)^{\prime}}\right)$, and it depends on the choices $\beta_{P}$ and $\beta_{Q}$. However, the composition

$$
\begin{aligned}
\operatorname{Isom}\left(\sigma_{P(n)}, \sigma_{Q(n)}\right) \approx & \operatorname{Isom}\left(\sigma_{\left(P^{n}\right)^{\prime}}, \sigma_{\left(Q^{n}\right)^{\prime}}\right) \\
\searrow & \downarrow \\
& \operatorname{Isom}\left(\sigma_{\left(P^{n}\right)^{\prime}}, \sigma_{\left(Q^{n}\right)^{\prime}}\right) \bmod \mathscr{S S}
\end{aligned}
$$

is well defined and is independent of (2.28). Let $R^{\prime}(n)=\beta_{P} R(n) \beta_{Q}^{-1}$ and $S^{\prime}(n)=\beta_{Q} S(n) \beta_{P}^{-1}$. Then $\left(P^{n}\right)^{\prime}=R^{\prime}(n) S^{\prime}(n)$ and $\left(Q^{n}\right)^{\prime}=S^{\prime}(n) R^{\prime}(n)$. Applying (2.30) takes the elementary $c(R(n), S(n)): X_{P(n)} \rightarrow X_{Q(n)}$ to

$$
c\left(R^{\prime}(n) ; S^{\prime}(n)\right): X_{\left(P^{n}\right)^{\prime}} \rightarrow X_{\left(Q^{n}\right)^{\prime}}
$$

To summarize progress so far, we have shown that starting with $\gamma(R, S) \in$ $\pi_{1}(R S(\mathscr{E}) ; P, Q)$ and going up, over, and back down into $\operatorname{Isom}\left(\sigma_{\left(P^{n}\right)^{\prime}}, \sigma_{\left(Q^{n}\right)^{\prime}}\right)$ $\bmod \mathscr{S} \mathscr{S}$ results in $c\left(R^{\prime}(n), S^{\prime}(n)\right)$.

On the other hand, going across into $\pi_{1}\left(R S(\overline{\mathscr{E}}) ; P^{n}, Q^{n}\right)$ takes $\gamma(R, S)$ to the path $\gamma\left(R, S P^{n-1}\right)$ corresponding to $\left(R, S P^{n-1}\right): P^{n} \rightarrow Q^{n}$. Then going up into $\pi_{1}\left(R S(\mathscr{E}) ;\left(P^{n}\right)^{\prime},\left(Q^{n}\right)^{\prime}\right) \bmod \mathscr{S} \mathscr{S}$ yields $\gamma\left(R^{\prime},\left(S P^{n-1}\right)^{\prime}\right)$ where $\left(R^{\prime},\left(S P^{n-1}\right)^{\prime}\right):\left(P^{n}\right)^{\prime} \rightarrow\left(Q^{n}\right)^{\prime}$ with the matrices $R^{\prime}$ and $\left(S P^{n-1}\right)^{\prime}$ coming from a choice of bijections

$$
p: P^{n} \approx R\left(S P^{n-1}\right) \text { and } q: Q^{n} \approx\left(S P^{n-1}\right) R
$$

as in $(2.5)$. We finally end up with the conjugacy $c\left(R^{\prime},\left(S P^{n-1}\right)^{\prime}\right)$ in

$$
\operatorname{Isom}\left(\sigma_{\left(P^{n}\right)^{\prime}}, \sigma_{\left(Q^{n}\right)^{\prime}}\right) \bmod \mathscr{S} \mathscr{S} \text {. }
$$

To complete the argument, we must show it is possible to make the choices (2.31) in such a way that

$$
R^{\prime}(n)=R^{\prime} \quad \text { and } \quad S^{\prime}(n)=\left(S P^{n-1}\right)^{\prime} .
$$

For each pair of indices $(k, j) \in \mathscr{T} \times \mathscr{S}$ choose a bijection $\eta$ from the set

$$
\left\{\left(k_{0}, i_{1}, \ldots, i_{n}\right) \mid k_{0}=k, i_{n}=j, S\left(k_{0}, i_{1}\right) P\left(i_{1}, i_{2}\right) \cdots P\left(i_{n-1}, i_{n}\right)=1\right\}
$$

to the set

$$
\left\{(k, \alpha, j) \mid 1 \leq \alpha \leq\left(S P^{n-1}\right)(k, j)\right\} .
$$

Now consider $(i, \alpha, j)$ where $1 \leq \alpha \leq P^{n}(i, j)$ and let $\left(i_{0}, i_{1}, \ldots, i_{n}\right)$ be the element of (2.24) mapping to it under $\beta_{P}$. Then we set

$$
p((i, \alpha, j))=((i, k),(k, \beta, j))
$$

where $k$ is the unique state with $R\left(i_{0}, k\right) S\left(k, i_{1}\right)=1$ and $\left(k, i_{1}, \ldots, i_{n}\right)$ maps to $(k, \beta, j)$ under $\eta$. Similarly, consider $(k, \alpha, l)$ where $1 \leq \alpha \leq Q^{n}(k, l)$ and let $\left(k_{0}, k_{1}, \ldots, k_{n}\right)$ be the element of (2.24) mapping to it under $\beta_{Q}$. Let $\left(i_{1}, \ldots, i_{n}\right)$ be the $n$-tuple determined by the condition

$$
S\left(k_{0}, i_{1}\right) R\left(i_{1}, k_{1}\right) S\left(k_{1}, i_{2}\right) \cdots S\left(k_{n-1}, i_{n}\right) R\left(i_{n}, k_{n}\right)=1 .
$$


Then let

$$
q((k, \alpha, l))=((k, \beta, j),(j, l))
$$

where $j$ is the unique state satisfying $S\left(k_{n-1}, j\right) R\left(j, k_{n}\right)=1$ (which implies $\left.i_{n}=j\right)$ and $(k, \beta, j)$ is the image of $\left(k, i_{1}, \ldots, i_{n}\right)$ under $\eta$. The verification that these choices of $p$ and $q$ for (2.31) lead to the equations (2.32) is straightforward.

\section{FOG AND LIFT}

Assume $\left(X_{A}, \sigma_{A}\right)$ is aperiodic. Let $P=P_{1}=\operatorname{Fix}\left(\sigma_{A}\right)$ and $n=|P|$. A special case of Williams' lifting problem (LIFT) asks whether

$$
\rho_{A}: \operatorname{Aut}\left(\sigma_{A}\right) \rightarrow \operatorname{Aut}(P) \approx \Sigma_{n}
$$

is always surjective. In this section we discuss a class of examples which show that if FOG is true, then LIFT is false. This means that at least one of the two main problems FOG or LIFT is false.

Let $\left(X_{A}, \sigma_{A}\right)$ satisfy the conditions

$$
\operatorname{det}(t I-A)=t^{m} \cdot p(t), \quad \text { where } m \geq 0 \text { and } p(t) \text { is irreducible, }
$$$$
\text { Let } P_{k}=P_{k}\left(\sigma_{A}\right)=\text { periodic points of } \sigma_{A} \text { of period exactly } k \text {. }
$$

Assume $n=\left|P_{1}\right| \geq 3$ and that $\left|P_{k}\right|=0$ for $2 \leq k \leq n$.

Proposition (3.4). IF FOG is true, then there are elements of $\operatorname{Aut}(P) \approx \Sigma_{n}$ which are not in the image of $\rho_{A}$.

Proof. Since $n \geq 3$, it suffices to show the image of $\rho_{A}$ is abelian. The argument of [BLR, 7.3] generalizes essentially verbatim under the hypotheses (3.2) and (3.3) to show that $\rho_{A}$ sends the subgroup of $\operatorname{Aut}\left(\sigma_{A}\right)$ generated by elements of finite order to zero. Moreover, we know $\operatorname{Aut}\left(s_{A}\right)$ is abelian from (3.2) and [BLR, 6.4]. Let $\delta_{A}$ denote the dimension group representation from $\operatorname{Aut}\left(\sigma_{A}\right)$ to $\operatorname{Aut}\left(s_{A}\right)$. If $x$ and $y$ lie in $\operatorname{Aut}\left(\sigma_{A}\right)$, then $\delta_{A}(x y)=\delta_{A}(x) \delta_{A}(y)=\delta_{A}(y) \delta_{A}(x)=$ $\delta_{A}(y x)$. FOG implies $x y=y x b$ for some $b$ in Aut $\left(\sigma_{A}\right)$ which is a product of finite order elements. Therefore, $\rho_{A}(x) \rho_{A}(y)=\rho_{A}(x y)=\rho_{A}(y x b)=$ $\rho_{A}(y x) \rho_{A}(b)=\rho_{A}(y) \rho_{A}(x)$.

Example (3.5). Let $A$ be the zero-one matrix corresponding to the graph in Figure 1 having $(n+1)$ vertices around a simple cycle and $n$ self-loops where $n \geq 3$. The matrix $A$ is aperiodic and clearly (3.3) holds. We claim (3.2) is valid as well and must show that the factor $\chi_{A}(t)^{x}$ of the characteristic polynomial corresponding to the nonzero eigenvalues is irreducible. In this case $\chi_{A}(t)^{x}=t(t-1)^{n}-1$. Emery Thomas kindly provided the following argument: Make the substitution $t=x+1$ to convert $\chi_{A}(t)^{x}$ to $x^{n+1}+x^{n}-1$. As zero is not a root, irreducibility in $Q[x]$ is equivalent to irreducibility in $Q\left[x, x^{-1}\right]$. Let $x=y^{-1}$ to get $x^{n+1}+x^{n}-1=-y^{-n-1}\left(y^{n+1}-y-1\right)$. It was shown in [S] that $y^{n+1}-y-1$ is irreducible. 


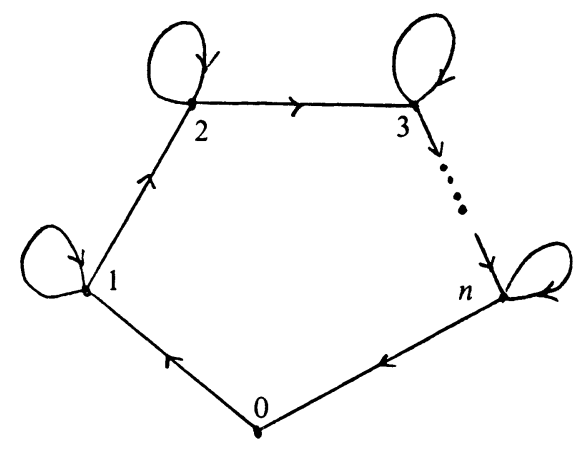

FigURe 1

Assuming FOG, Brian Marcus pointed out more specifically in these examples that none of the elements of $\operatorname{Aut}(P) \approx \Sigma_{n}$ lying outside the abelian subgroup generated by the cyclic permutation $\beta=(123 \cdots n)$ are in the image of $\rho_{A}$. Here is his argument. Consider the zero-one matrix $B$ arising from the graph in Figure 2 having $n+1$ vertices. Then $B^{n}=A$. This implies $\sigma_{B^{n}}=\sigma_{A}$ and $\sigma_{B}$ belongs to $\operatorname{Aut}\left(\sigma_{A}\right)$. The image of $\sigma_{B}$ in $\operatorname{Aut}(P)$ is $\beta^{-1}$, and the image of $\rho_{A}$ is abelian. Therefore, the image of $\rho_{A}$ is precisely the subgroup generated by $\beta$, because no other elements in $\Sigma_{n}$ commute with $\beta$.

Using his deep result on gyration numbers [F], Fiebig can show the truth of FOG in general would imply there are examples where

$$
\operatorname{Aut}\left(\sigma_{n}\right) \rightarrow \operatorname{Aut}\left(\sigma_{n} \mid \operatorname{Fix}\left(\sigma_{n^{k}}\right)\right)^{2}
$$

is not onto for certain $n$ and certain $k>1$.

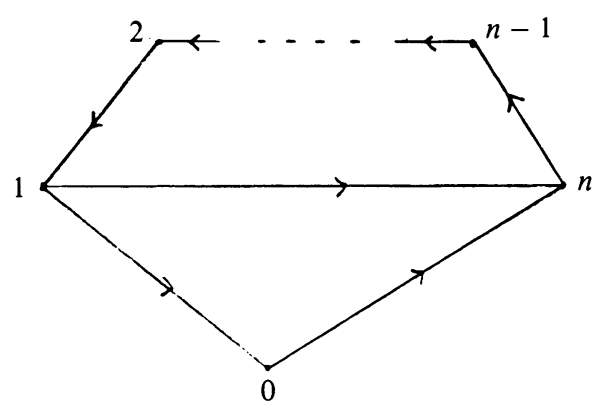

FIGURE 2

\section{Stable FOG and gyration Numbers}

Consider the full gyration number homomorphism

$$
\mathrm{gy}_{A}: \operatorname{Aut}\left(\sigma_{A}\right) \rightarrow \prod_{m \geq 2} Z / m
$$

\footnotetext{
${ }^{2}$ Kim-Roush [KR] have shown this is not surjective for $n=2$ and $k=6$.
} 
which is the product of the individual gyration number homomorphisms $\mathrm{gy}_{A, m}: \operatorname{Aut}\left(\sigma_{A}\right) \rightarrow Z / m$. This was introduced in [BK] and further studied in [BLR, F]. The left-hand side is countable whereas the right-hand side is uncountable, and therefore $\mathrm{gy}_{A}$ is not onto. We will show in (4.11) that, for example, the sequence $(1,1, \ldots)$ does not belong to the image of the full gyration number homomorphism $\mathrm{gy}_{n}$ for the Bernoulli $n$-shift $\sigma_{n}$.

Proposition (4.1). Let $\alpha \in \operatorname{Aut}\left(\sigma_{A}\right)$ lie in the kernel of the dimension group representation $\delta_{A}$. Let $p$ be a rational prime. The gyration number of $\alpha$ for $m=p^{k}$ converges $p$-adically to zero as $k \rightarrow \infty$.

Corollary (4.2). Let $p$ be a rational prime. For $\alpha \in \operatorname{Aut}\left(\sigma_{n}\right)$ the gyration number of $\alpha$ for $m=p^{k}$ converges $p$-adically as $k \rightarrow \infty$.

These results give a partial answer to Question 8.5 of [BLR].

Proof of (4.1). Assume $u=p^{n}$ and $v=p^{k}$ where $p$ is prime. From [BK, 1.5] we have $P_{u v}\left(\sigma_{A}\right)=P_{v}\left(\sigma_{A^{u}}\right)$ and a commutative square:

$$
\begin{array}{ccc}
\operatorname{Aut}\left(\sigma_{A}\right) & \rightarrow & \operatorname{Aut}\left(\sigma_{A} \mid P_{u v}\left(\sigma_{A}\right)\right) \\
\downarrow & & \downarrow \\
\operatorname{Aut}\left(\sigma_{A^{u}}\right) & \rightarrow & \operatorname{Aut}\left(\sigma_{A^{u}} \mid P_{v}\left(\sigma_{A^{u}}\right)\right)
\end{array}
$$

Moreover, from [BK, 1.6] we know that there is a commutative diagram

$$
\begin{array}{ccc}
\operatorname{Aut}\left(\sigma_{A} \mid P_{u v}\left(\sigma_{A}\right)\right) & \underset{\mathrm{gy}_{A, u v}}{\rightarrow} & Z / u v \\
\downarrow & \downarrow \\
\operatorname{Aut}\left(\sigma_{A^{u}} \mid P_{v}\left(\sigma_{A^{u}}\right)\right) & \underset{\mathrm{gy}_{A} u_{, v}}{\rightarrow} & Z / v
\end{array}
$$

where $Z / u v \rightarrow Z / v$ is the standard homomorphism.

Now let $\alpha$ be in the kernel of $\delta_{A}$ and let $k_{0}$ be the integer provided by (1.1) so that for each $q \geq k_{0}, \alpha$ is a product of involutions commuting with $\sigma_{A^{q}}$. Fix $u=p^{n} \geq k_{0}$ and let $v=p^{k}$. Since $\alpha$ is a product of involutions commuting with $\sigma_{A^{u}},(4.3)$ implies the order of $\mathrm{gy}_{A, u v}(\alpha)$ considered as lying in the abelian group $Z / v$ divides 2 . Therefore $2 \mathrm{gy}_{A, u v}(\alpha)$ lies in the kernel $Z / u$ of $Z / u v \rightarrow Z / v$. So we have $2 u \mathrm{gy}_{A, u v}(\alpha)=0$ in $Z / u v$. If $p=2$, this implies $p^{k-1}$ divides the order of $\operatorname{gy}_{A, u v}(\alpha)$. If $p$ is odd, this implies $p^{k}$ divides the order of $\mathrm{gy}_{A, u v}(\alpha)$. Hence $\mathrm{gy}_{A, u v}(\alpha)$ goes to zero $p$-adically as $v \rightarrow \infty$.

Proof of (4.2). The dimension group of the full shift $\sigma_{n}$ is isomorphic to $Z[1 / n]$, and its automorphism group $\operatorname{Aut}\left(s_{n}\right)$ is the positive invertible elements in the ring $Z[1 / n]$. This is the multiplicative free abelian group generated by the distinct rational primes $r$ dividing $n$. Let $r s=n$. Then $\sigma_{n}=\sigma_{r} \times \sigma_{s}$ and from [W1] we know the symmetry $\sigma_{r} \times 1$ of $\sigma_{n}$ maps to $r$ under $\delta_{n}$. The proof of (8.2) in [BLR] shows that

$$
\begin{aligned}
& \lim _{k} \operatorname{gy}_{n, p^{k}}\left(\sigma_{r} \times 1\right)=0 \quad \text { if } p \text { divides } r \text { or } s, \\
& \lim _{k} \operatorname{gy}_{n, p^{k}}\left(\sigma_{r} \times 1\right)=(1-1 / p) \omega(n) \log _{p}(r) \quad \text { if }(p, r)=(p, s)=1,
\end{aligned}
$$


where the $(p-1)$ th root of unity $\omega(n)$ is the Teichmüller representative of $n$ in $Q_{p}$. Let $D(n)$ denote the subgroup of $\operatorname{Aut}\left(\sigma_{n}\right)$ generated by the various $\sigma_{r} \times 1$. The map $\delta_{n}: D(n) \rightarrow \operatorname{Aut}\left(s_{n}\right)$ is surjective, and therefore $\operatorname{Aut}\left(\sigma_{n}\right)$ is generated by $D(n)$ and $\operatorname{Ker}\left(\delta_{n}\right)$. Write $\alpha \in \operatorname{Aut}\left(\sigma_{n}\right)$ as a product $\alpha=\beta \gamma$ with $\beta \in D(n)$ and $\gamma \in \operatorname{Ker}\left(\delta_{n}\right)$. Then

$$
\operatorname{gy}_{n, p^{k}}(\alpha)=\operatorname{gy}_{n, p^{k}}(\beta)+\operatorname{gy}_{n, p^{k}}(\gamma) \text {. }
$$

As $k \rightarrow \infty$ the first term on the right-hand side converges by (4.4) and the second converges (to zero) by (4.1). Therefore the left-hand side converges as $k \rightarrow \infty$.

From (4.2) and the above discussion we now obtain a homomorphism

$$
\operatorname{gy}_{n, p}: \operatorname{Aut}\left(\sigma_{n}\right) \rightarrow \mathbf{Q}_{p}
$$

into the additive group $\mathbf{Q}_{p}$ defined by

$$
\operatorname{gy}_{n, p}(\alpha)=\lim _{k} \mathrm{gy}_{n, p^{k}}(\alpha) .
$$

Proposition 4.1 implies that

$$
\operatorname{gy}_{n, p \infty}\left(\operatorname{Aut}\left(\sigma_{n}\right)\right)=\operatorname{gy}_{n, p^{\infty}}(D(n)) \text {. }
$$

Corollary (4.8). The image of $\mathrm{gy}_{n, p \infty}$ is zero if $p$ divides $n$. If $p$ and $n$ are relatively prime, then the image of $\mathrm{gy}_{n, p^{\infty}}$ is the free abelian group of integral linear combinations of the values $(1-1 / p) \omega(n) \log _{p}(r)$ where $r$ runs over the distinct primes dividing $n$.

Linear independence of the values $(1-1 / p) \omega(n) \log _{p}(r)$ over $\mathbf{Z}$ comes from the $p$-adic version [Br] of Baker's transcendence results in homogeneous from [B, (I) and (II)].

Let $\left\{\nu_{2}, \nu_{3}, \ldots\right\}$ be a sequence of integers where we consider $\nu_{m}$ as lying in $Z / m$. Assume for some prime $p$ that

$$
\begin{aligned}
& \text { either } \nu=\lim \nu_{p^{k}} \text { does not exist in } \mathbf{Q}_{p} \text {, or the limit } \nu \text { does } \\
& \text { exist and is a nonzero algebraic number over } \mathbf{Q} \text {. }
\end{aligned}
$$

Corollary (4.10). The sequence $\left\{\nu_{2}, \nu_{3}, \ldots\right\}$ is not in the image of $\mathrm{gy}_{n}$.

Proof. If the sequence $\left\{\nu_{2}, \nu_{3}, \ldots\right\}$ is in the image of $\mathrm{gy}_{n}$, the limit must exist by (4.2) for all primes $p$. So suppose $\nu$ exists and is a nonzero algebraic number over $\mathbf{Q}$. Suppose $\left\{\nu_{2}, \nu_{3}, \ldots\right\}$ is in the image. Then $\nu$ would be a linear combination with algebraic number coefficients of various $\log _{p}(r)$ in contradiction to the $p$-adic version [W, Theorem 5 of Appendix I by D. Bertrand] of Baker's transcendence results in nonhomogeneous form [B, (III)].

Here is a more elementary result. 
Proposition (4.11). Let $q$ be a nonzero integer and assume $n$ is prime. The constant sequence $\{q, q, \ldots\}$ is not in the image of $\mathrm{gy}_{n}$.

Proof. The group $D(n)$ is generated by $\sigma_{n}$. Choose a prime $p$ not equal to $n$. If $\{q, q, \ldots\}$ were in the image, then we would have $k(1-1 / p) \omega(n) \log _{p}(n)=$ $q$ for some $k \neq 0$, contradicting the fact that $\log _{p}(n)$ is transcendental.

Recent work of Kim-Roush [KR] combined with [F] now gives much more information on gyration number sequences.

\section{REFERENCES}

[B] A. Baker, Linear forms in the logarithms of algebraic numbers (I), (II), (III), Mathematika 13, 14 (1966-67).

[Br] A. Brumer, On the units of algebraic number fields, Mathematika 14 (1967), 121-124.

[Boy] M. Boyle, Eventual extension of finite codes, Proc. Amer. Math. Soc. 104 (1988), 965-971.

[BFK] M. Boyle, J. Franks, and B. Kitchens, Automorphisms of one sided subshifts of finite type, preprint, IBM Research, T. J. Watson Research Center, Yorktown Heights, 1988; Eergodic Theory Dynamical Systems (to appear).

[BK] M. Boyle and W. Krieger, Periodic points and automorphisms of the shift, Trans. Amer. Math. Soc. 302 (1987), 125-149.

[BLR] M. Boyle, D. Lind, and D. Rudolph, The automorphism group of a subshift of finite type, Trans. Amer. Math. Soc. 306 (1988), 71-114.

[F] U. Fiebig, Gyration numbers for involutions of subshifts of finite type, preprint, University of Heidelberg, 1988.

[KR] K. H. Kim and F. W. Roush, On the structue of inert automorphisms of subshifts, preprint, Alabama State University, March 1989.

[N] M. Nasu, Topological conjugacy of sofic systems and extensions of automorphisms of finite subsystems of topological Markov shifts, Proceedings of the Special Year in Dynamical Systems at the University of Maryland 1986-87, Lecture Notes in Math., vol. 1342, Springer-Verlag, 1988.

[S] E. S. Selmer, On irreducibility of certain trinomials, Math. Scand. 4 (1956), 287-302.

[W] M. Waldschmidt, Nombres transcendants et groupes algebriques, Theorem 5 of Appendix I by D. Bertrand, Asterisque Nos. 69-70, Soc. Math. de France, 1979.

[W1] J. B. Wagoner, Markov partitions and $K_{2}$, Inst. Hautes Études Sci. Publ. Math. 65 (1987), 91-129.

[W2] _ Triangle identities and symmetries of a subshift of finite type, preprint, Univ. of California, Berkeley, 1987; Pacific J. Math. (to appear).

Department of Mathematics, University of California, Berkeley, California 94720 